\title{
Search for intra-day optical variability in Mrk 501
}

\author{
Hai-Cheng Feng ${ }^{1,2,3}$, H. T. Liu ${ }^{1,3,4}$, X. L. Fan ${ }^{1,2,3}$, Yinghe Zhao ${ }^{1,3,4}$, J. M. Bai ${ }^{1,3,4}$, Fang \\ Wang $^{1,2,3}$, D. R. Xiong ${ }^{1,2,3}$, and S. K. Li ${ }^{1,3,4}$
}

Received __; accepted _

\footnotetext{
${ }^{1}$ Yunnan Observatories, Chinese Academy of Sciences, 396 Yangfangwang, Guandu District, Kunming, 650216, P. R. China

${ }^{2}$ University of Chinese Academy of Sciences, Beijing 100049, P. R. China

${ }^{3}$ Key Laboratory for the Structure and Evolution of Celestial Objects, Chinese Academy of Sciences, 396 Yangfangwang, Guandu District, Kunming, 650216, P. R. China

${ }^{4}$ Center for Astronomical Mega-Science, Chinese Academy of Sciences, 20A Datun Road, Chaoyang District, Beijing, 100012, P. R. China

${ }^{\star}$ Corresponding author: H. T. Liu, e-mail: htliu@ynao.ac.cn
} 


\begin{abstract}
We present our observations of the optical intra-day variability (IDV) in $\gamma$-ray BL Lac object Mrk 501. The observations were run with the $1.02 \mathrm{~m}$ and $2.4 \mathrm{~m}$ optical telescopes at Yunnan Observatories from 2005 April to 2012 May. The light curve at the $R$ band on 2010 May 15 passes both variability tests (the $F$ test and the ANOVA test). A flare within the light curve on 2010 May 15 has a magnitude change $\Delta m=0.03 \pm 0.005_{\text {stat }} \pm 0.007_{\text {sys }}$ mag, a darkening timescale of $\tau_{\mathrm{d}}=\mathbf{2 6 . 7}$ minutes, and an amplitude of IDV $A m p=2.9 \% \pm 0.7 \%$. A decline described by 11 consecutive flux measurements within the flare can be fitted linearly with a Pearson's correlation coefficient $r=0.945$ at the confidence level of $>99.99 \%$. Under the assumptions that the IDV is tightly connected to the mass of the black hole, and that the flare duration, being two times $\tau_{\mathrm{d}}$, is representative of the minimum characteristic timescale, we can derive upper bounds to the mass of the black hole. In the case of the Kerr black hole, the timescale of $\Delta t_{\mathrm{min}}^{\mathrm{ob}}=0.89$ hours gives $M_{\bullet} \lesssim 10^{9.20} M_{\odot}$, which is consistent with measurements reported in the literature. This agreement indicates that the hypothesis about $M_{\bullet}$ and $\Delta t_{\min }^{\mathrm{ob}}$ is consistent with the measurements/data.
\end{abstract}

Subject headings: galaxies: active — galaxies: nuclei — BL Lacertae objects: individual (Mrk 501) 


\section{INTRODUCTION}

Blazars are the most violently variable subclass of active galactic nuclei (AGNs), which include BL Lacertae objects (BL Lacs) and flat spectrum radio quasars (FSRQs). They are characterized by rapid and strong variability at all wavelengths of the whole electromagnetic spectrum, strong and variable polarization from radio to optical bands, non-thermal emission dominating at all wavelengths. Blazars usually exhibit the core-dominated radio shape. These extreme properties are generally believed to be generated from a relativistic jet with a viewing angle less than $10^{\circ}$ (e.g., Blandford \& Königl 1979; Urry \& Padovani 1995). The broadband spectral energy distributions (SEDs) of blazars usually show a double-peak profile. The low-energy component spans the IR-optical-UV bands and the high-energy component extends to GeV/TeV gamma-ray bands (e.g., Ghisellini et al. 1998; Abdo et al. 2010a). The first peak is generally interpreted as the synchrotron radiation of relativistic electrons in the jet. The second peak is generally believed to be generated by the inverse-Compton scattering of the same electron distribution responsible for the synchrotron radiation (e.g., Dermer \& Schlickeiser 1993; Böttcher 2007; Neronov et al. 2012).

Photometric technique is a powerful tool to investigate the nature of blazars. The variability including amplitude, duty cycles, timescales and so on, could help us to study the structure, dynamics, radiation mechanism and even phase of blazars (e.g., Ciprini et al. 2003, 2007; Gupta et al. 2008a; Dai et al. 2015; Xiong et al. 2015). Previous observations show that blazars exhibit variability on diverse timescales, which can be broadly divided into three classes: intra-day variability (IDV) or micro-variability, short-term variability (STV), and long-term variability (LTV). For IDVs, the timescales range from a few minutes to several hours, and the variability of flux changes by a few tenths of magnitude (e.g., Wagner \& Witzel 1995). STVs and LTVs have timescales of days to months and months 
to years, respectively (e.g., Fan 2005; Fan et al. 2009; Gupta et al. 2008b; Dai et al. 2015; Xiong et al. 2015). Over the last three decades, a great mount of work for variability of blazars has been reported at different timescales in different bands (e.g., Miller et al. 1989; Bai et al. 1998; Catanese \& Sambruna 2000; Xie et al. 1990, 1991a,b, 1992, 1999, 2001, 2002b.c, 2005a; Fan 2005; Fan et al. 2009; Aharonian et al. 2007; Ciprini et al. 2003, 2007; Gupta et al. 2004, 2008a,bi ; Dai et al. 2015; Xiong et al. 2015; Ahnen et al. 2016). The IDVs of the beamed emission of jets can be used to constrain the central black hole masses of blazars (Liu \& Bai 2015).

Mrk 501 is a typical nearby BL Lac object (at redshift $z=0.034$ ), one of the nearest Jy BL Lac objects (Stickel et al. 1993; Konopelko et al. 2003) and one of the brightest extragalactic sources in the X-ray/TeV sky (Abdo et al. 2011). It has attracted much attention. Since the first detection by Whipple observatory (Quinn et al. 1996), Mrk 501 was the second extragalactic object identified as a very high energy (VHE) $\gamma$-ray emitter (Konopelko et al. 2003; Abdo et al. 2011; Xiong et al. 2015). In the second year since it was discovered, Mrk 501 went into a surprisingly high activity and strong variability state. Fluxes over 10 times brighter than 10 Crab were reported in several groups (Catanese et al. 1997; Samuelson et al. 1998; Aharonian et al. 1999a,b). After the outburst state, the mean VHE $\gamma$-rays dropped by an order of magnitude during 1998-1999 (Aharonian et al. 2001). In 2005, an interesting phenomenon, the VHE $\gamma$-ray flux varies on a timescale of minutes, was detected (Albert et al. 2007). The recent report about a multi-wavelength study in 2009 shows fast variability with $\sim 15$ minute doubling times in the VHE gamma rays (Ahnen et al. 2016). The variability of Mrk 501 on the whole electromagnetic wavelengths has been extensively studied (e.g., Xie et al. 1999, 2001; Gupta et al. 2008b; Rodig et al. 2009; Gupta et al. 2012; Aleksic et al. 2015; Shukla et al. 2015; Wierzcholska et al. 2015; Xiong et al. 2015; Ahnen et al. 2016). The IDVs of Mrk 501 have been reported over the entire electromagnetic spectrum (e.g., Albert et al. 2007; 
Gupta et al. 2008b; Shukla et al. 2015; Xiong et al. 2015). Most of previous works were on timescales of tens to one hundred minutes for the optical IDVs. A rapid X-ray flare from Mrk 501 detected with the Rossi X-Ray Timing Explorer in 1998 shows the $60 \%$ increase in 200 seconds ( $\sim 3$ minutes) (Catanese \& Sambruna 2000). Xie et al. (1999) reported a $R$ band flare on a timescale of 105 minutes. Xiong et al. (2015) found a $R$ band flare around 106 minutes. Compared to the X-ray and VHE flares on timescales of minutes, the optical IDVs are on timescales of hours. Shorter optical IDVs may be expected.

In this paper, we present observations and investigate the variability in $B, I, R$, and $V$ bands from 2005 to 2012. To find out the fastest optical IDV, most exposure times are less than 3 minutes. The structure of this paper is as follows: in section 2 we present the observations and data reduction; the results of variability are exhibited in section 3; section 4 is for discussion.

\section{OBSERVATIONS AND DATA REDUCTION}

The optical monitoring program of Mrk 501 was carried out with two telescopes: the $1.02 \mathrm{~m}$ and $2.4 \mathrm{~m}$ optical telescopes at Yunnan Observatories, China. The $1.02 \mathrm{~m}$ telescope is located at Kunming, China. From 2006 to 2009, the telescope equipped with a RAC CCD (1024 pixels $\times 1024$ pixels) at $f / 13.3$ Cassegrain focus, and the entire CCD chip covered $\sim 6.5 \times 6.5 \operatorname{arcmin}^{2}$. So, the projected angle on the sky of each pixel corresponded to 0.38 arcsec in both dimensions. The readout noise and gain were 3.9 electrons and 4.0 electrons/ADU, respectively. After 2009, the telescope was equipped with an Andor AW436 CCD (2048 pixels $\times 2048$ pixels) camera at the $f / 13.3$ Cassergrain focus. The field of view of the CCD is $\sim 7.3 \times 7.3 \operatorname{arcmin}^{2}$, and the pixel scale is 0.21 arcsec in both dimensions. The readout noise and gain were 6.33 electrons and 2.0 electrons/ADU, respectively (e.g., Xie et al. 2002b; Gupta et al. 2008a; Dai et al. 2015). The $2.4 \mathrm{~m}$ telescope is located at 
Lijiang, China. The telescope was equipped with an Princeton Instruments VersArray1300B CCD (1300 pixels $\times 1340$ pixels) camera at f/8 Cassegrain focus, and the entire CCD chip covered $\sim 4.40 \times 4.48 \operatorname{arcmin}^{2}$. The readout noise and gain were 6.5 electrons and 1.1 electrons/ADU, respectively. For both of the two telescopes, we selected standard Johnson broadband filters to carry out the observations in the $B, V, R$, and $I$ bands.

During our monitoring program from 2005 to 2012, 1532 CCD frames were obtained in 50 nights. For most nights, the exposure times are 40-400 seconds for $1.02 \mathrm{~m}$ telescope and 10-50 seconds for $2.4 \mathrm{~m}$ telescope. The complete observation log is listed in Table 1. For each image, the standard stars and object were always in the same field. The standard stars considered do not change, so the brightness of the object was obtained from the standard stars (e.g., Bai et al. 1998; Fan et al. 2014; Zhang et al. 2004, 2008). Because star 1 is the brightest of all comparison stars and the magnitudes have been measured in all bands in other works (Villata et al. 1998; Fiorucci \& Tosti 1996), it was selected to calculate the object magnitude. However, there are some uncertainties which may cause the standard stars to change, so we chose another standard star to characterize the change, and we used the standard deviation of the two comparison stars $[\sigma(\operatorname{star} 1-s t a r 6)]$ as the error of photometry. Star 6, closest to the object and with all four bands measured at the same time with star 1 , is used as the second standard star. The standard deviation of the differential instrumental magnitude of star1-star6 is given in Table 1. The IDV of the target object was investigated using two statistical methods: the $F$ test (e.g., de Diego et al. 2010; Joshi et al. 2011; Goval et al. 2012; Hu et al. 2014; Agarwal \& Gupta 2015) and the one-way analysis of variance (ANOVA; de Diego et al. 1998, 2010; Hu et al. 2014). The value of $F$ test in our work is calculated as:

$$
F=\frac{\operatorname{Var}(B L-\operatorname{star} 1)}{\operatorname{Var}(\operatorname{star} 1-\operatorname{star} 6)}
$$

where BL, star1, and star6 are the magnitudes of BL Lac object, star1, and star6, 
respectively. $\operatorname{Var}(B L-s t a r 1)$ and $\operatorname{Var}($ star1-star6) are the variances of differential instrumental magnitudes for 'BL-star1' and 'star1-star6', respectively. The critical value of the $F$ test can be compared with the $F$ value for which the significance level was set as 0.01 in $F$-statistics. ANOVA is a powerful tool to detect IDVs, and does not rely on error measurements. We divided data points into groups. Each group contains three data points, and if the last group has less than three data points, we would merger them with the previous group. The ANOVA critical value can be obtained from $F_{\nu 1, \nu 2}^{\alpha}$ in the F-statistics, where $\nu 1$ ( $\nu 1=k-1$, and $k$ is the number of groups) is the degree of freedom between groups and $\nu 2(\nu 2=N-k$, and $N$ is the number of measurements $)$ is the degree of freedom within these groups, and $\alpha$ is the significance level (e.g., Hu et al. 2014). When calculating the values of $F$ and ANOVA, we only used the light curves with observational data points $\geq 9$ in a night, and only the light curves satisfied with both criteria were considered to be variable. Table 1 shows the results of $F$ test and ANOVA for all the observable nights satisfying the criteria.

All of the photometric data were reduced using the standard procedure in the Image Reduction and Analysis Facility (IRAF) software. For each night, we combined all the bias frames and then obtained a master bias. All of the object image frames and flat-field image frames were subtracted by the master bias. Then we generated the master flat-field for each band by taking the median of all flat-field image frames for each band. After the flat-field correction, aperture photometry was performed by the APPHOT task. Because Mrk 501 is an extended source and the standard stars are point like sources, we used two different criteria to determine the aperture size for the object and comparison stars. For the standard stars, we found that the best signal-to-noise ratio was obtained with the aperture radius of 1.2 full width at half maximum (FWHM), i.e. a dynamic aperture, which is generally applied to the point like sources consisting of AGNs and their host galaxies, and is large enough to cover the hosts. For extended sources, such as nearby AGNs, the 
change of seeing may affect the dynamic aperture and then the contribution of the hosts. If the host is considered to be constant for Mrk 501, the photometric results could reflect a combined contribution of the aperture effect and the intrinsic variations of AGN. We chose 16 different aperture radii among 1.2-8.0 FWHMs, and the variability of Mrk 501 is similar to each other when the apertures are greater than or equal to than 3 FWHMs (see the upper left panel in Figure 1). The light curves with apertures of 6.0-8.0 FWHMs have almost the same outline, i.e., the light curve profile is stable as the aperture is enough large. It seems that there is an arc dip on 2010 May 17 lasting for about 90 minutes. However, the aperture is dynamic with the FWHM of standard star, and the light curve profile may be biased by the FWHM variability. The lower left panel in Figure 1 shows the FWHM variability. This FWHM variability is very similar to those of the light curves in 1.2-2.5 FWHMs, especially the arc dip. The smaller FWHM results in the smaller aperture, and the fewer host contribution. This will result in the darkening of Mrk 501. A flare in the $I$ band on 2009 May 11 has a large magnitude change of $\Delta I=0^{m} .28$ and a symmetrical profile, when the dynamical aperture is used. However, the corresponding FWHMs nearly have the same variability as the light curve of Mrk 501 (see Figure 1). Thus, the dynamical aperture significantly influences the variability results of the photometry due to the FWHM variability, and the fixed aperture is used to perform photometry. The fixed aperture can avoid this host influence on the measured magnitude variability of Mrk 501. In order to contain the most contribution of AGN and avoid the effect of background, we used 3.5 arcsec aperture radius, which is more than $1.5 \times$ typical FWHM for most of images.

Though the fixed aperture can avoid the host influence of the dynamic aperture on the variability of Mrk 501, the seeing effect influences the FWHM, and then the fraction of the host light contained by the fixed aperture. Because the profile of the host galaxy is not flat Nilsson et al. (2007), the different point spread functions influence the contribution of the host in a fixed aperture. However, this influence is much smaller than that of the 
dynamic aperture mentioned above. For this host, the larger seeing results in the larger point spread function that leads to the smaller faction of the host light contained by the fixed aperture. The increasing FWHM will generate the darkening of measured magnitude. The observational data and the fit results confirm the deduced FWHM-magnitude relation (see Figure 2). From 2010 May 15 to 2010 May 18, four night observations are used to test this relation. Also, there is a similar relation for these four night data. Thus, the FWHM-magnitude relation is corrected for each night data. The host contribution is subtracted according to the method of Nilsson et al. (2007) for the data on 2010 May 18, and the FWHM-magnitude relation appears more significant. The host-corrected magnitudes depend on point spread function or seeing (FWHM). This FWHM-magnitude relation is corrected to get the final result for each night data. Figure 3 shows the light curves in the $I$ band on 2009 May 11 for a fixed aperture without and with correcting the FWHM-magnitude relation. Comparison of the light curves in Figure 3 shows that the seeing effect is improved. The IDV seems to exist in the corrected light curve in Figure 3. The $F$ test or the ANOVA test show six possible IDVs, and only the IDV on 2010 May 15 is confirmed by the two tests (see Table 1). Two interesting light curves with the possible IDVs are presented in Figure 4.

\section{RESULTS}

Table 1 shows six nights for which the the light curve gave positive in the $F$ or ANOVA tests, from which only one night, 2010 May 15, gave positive in both tests. As an example, Figure 4 shows two nights, 2010 May 15 and 17. These light curves have a peak-to-peak flux change of $\Delta R \sim 0.03 \mathrm{mag}$, while the observational error estimated by the standard deviation from the magnitude differences between standard star 1 and star 6 is $\sigma_{\mathrm{R}}=0.005$.

Therefore, the peak-to-peak flux change $\Delta R \sim 6 \sigma_{\mathrm{R}}$. We followed the method in Du et al. 
(2014) to determine the systematic error of the fluxes. First, we used a median filter to smooth the light curve, and then subtracted it from the original light curve. Second, we calculated the standard deviation of the residual light curve, and adopted this deviation as an estimate of the systematic uncertainty. The value estimated with this method is 0.007 mag. If this systematic error is combined quadratically with the statistical error, we find a total uncertainty in the flux variation of $\sigma_{T}=0.009$, which leads to a peak-to-peak flux change of $\Delta R \sim 3.3 \sigma_{T}$. The detection success rate of the optical IDVs seems to be very low for Mrk 501. This may be due to the intrinsic weak IDVs of Mrk 501 and/or the relatively brighter host galaxy. For the large amplitude variations of BL Lacertae objects, an effective variation on a short timescale (from a few hundred seconds to several hours) requires that the amplitude of optical variability must be more than $5 \sigma$, where $\sigma$ is the maximum total observational rms error (Xie et al. 1990, 1992, 2004). The rms error used in Xie et al. (2004) is the same as the standard deviation of the two comparison stars we used in this paper. The ratio of $\Delta m / \sigma>5$ (where $\sigma$ relates to the statistical uncertainty) was used as a necessary criterion rather than a sufficient and necessary condition for optical variability. Though, the light curves on 2010 May 15 and 17 match the criterion $\Delta m>5 \sigma$, they do not necessarily have IDVs because of the systematic uncertainties mentioned above.

The long-term light curves are presented in Figure 5 for our observational data. The larger amplitudes of variability are found in the long-term light curves. The continuous observations in the light curves appear to be a cluster, which may show a larger amplitude of variability. A variability amplitude of $\Delta B \sim 0.8 \mathrm{mag}$ is around $\mathrm{MJD}=54900$ for the $B$ band. We find a variability amplitude of $\Delta I \sim 0.7$ mag for the $I$ band around $\mathrm{MJD}=55250$. The long-term light curve in the $R$ band is similar to that in the $I$ band. However, the $R$ band FWHMs are larger than 5.3 arcseconds on 2006 April 3, which may be due a worse observational condition. The $I$ band FWHMs are smaller than those of the $R$ 
band on 2006 April 3, and the $I$ band observations were completed before the $R$ band observations. This may result in a worse photometric magnitude in the $R$ band, darker than 18 mag (see Figure 5). The other data points of the $R$ band are not particularly surprising. There is a variability amplitude of $\Delta R \sim 0.7 \mathrm{mag}$ around MJD=55250. For the $V$ band around $\mathrm{MJD}=54200$, a variability is found with an amplitude of $\Delta V \sim 0.7$ mag. Also, a variability is found with an amplitude of $\Delta V \sim 0.7$ mag around MJD=56000. These variations with large amplitudes of $\Delta m \sim 0.7-0.8$ mag have durations within $\sim 100$ days. Also, the similar timescale variations are found in Seyfert galaxies, e.g., NGC 5548 (Ulrich et al. 1997). These timescales within $\sim 100$ days may be from the lighthouse effect of a jet, where the jet precession will result in the forward beaming of the emission (e.g., Camenzind \& Krockenberger 1992; Gopal-Krishna \& Wiita 1992).

\section{DISCUSSION}

Except for the jet origin of the optical variations, there is an alternative way to explain the optical variability and the IDVs or micro-variability in BL Lac objects, i.e. the optical IDVs are likely from accretion disks (e.g., Gupta et al. 2012; Agarwal et al. 2016). The accretion disk instabilities are able to explain some of the phenomena seen in the optical-X-ray bands, but cannot explain the radio IDVs (e.g., Wagner \& Witzel 1995). The latest research on BL Lac object PKS $0735+178$ shows that the blazar variability on timescales from years down to hours-i.e., both the long-term large amplitude variability and the micro-variability-is generated by the underlying single stochastic process (at the radio and optical bands), or a linear superposition of such processes (in the gamma-ray regime), within a highly non-uniform portion of the flow extending from the jet base up to the $\lesssim$ pc-scale distances (Goyal et al. 2017). Impiombato et al. (2011) searched for short timescale variability, and identified an interesting event in the $J$ band with a duration of $\sim$ 
25 minutes for BL Lac object PKS 0537-441. In both the low and high states, the emission appears to be dominated by the jet, and no evidence of a thermal component is apparent for PKS 0537-441. The spectral energy distributions of PKS 0537-441 are interpreted in terms of the synchrotron and inverse Compton mechanisms within a jet, where the plasma radiates via internal shocks and the dissipation depends on the distance of the emitting region from the central engine (Pian et al. 2007). The optical emission of Mrk 501 is neither the thermal component from accretion disk nor the nonthermal component from the jet (Ahnen et al. 2016). The optical emission is dominated by the host galaxy, and the UV emission is from the jet for Mrk 501 (e.g., Ahnen et al. 2016). Thus, it is not possible that the optical IDVs are from accretion disk for Mrk 501.

The IDVs discussed here may be directly related to shock processes in a jet. The shock-in-jet model, the most frequently cited model used to explain the IDVs, is based on a relativistic shock propagating down a jet and interacting with a highly non-uniform portion in the jet flow (e.g., Narayan \& Piran 2012; Subramanian et al. 2012; Marscher 2014; Saito et al. 2015, and references therein). The featureless optical continuum is the typical characteristic of BL Lac objects, and quasars show many strong broad emission lines. The broad-line region (BLR) seems to not be in BL Lac objects (e.g., Urry \& Padovani 1995). Broad emission lines were observed only in a few BL Lac objects (e.g., Cao \& Jiang 1999; Celotti et al. 1997). Thus, the broad emission lines seem to be weak so that the broad lines were rarely observed in BL Lac objects. The accretion rates are very low for BL Lac objects (e.g., Cao 2002; Xu et al. 2009). The absence of broad emission lines in most of BL Lac objects may be due to the very weak emission of accretion disk. The BLR clouds are right there though observations have not detected the broad lines in most of BL Lac objects. Thus, the optical variability in BL Lac objects is not dominated by the emission of accretion disk. On the contrary, the relativistic jets are likely dominating the optical variability. Though a minority of BL Lac objects have the broad emission lines, they have 
Full width at half maximum $v_{\text {FWHM }} \sim 1300-5500 \mathrm{~km} \mathrm{~s}^{-1}(\mathrm{CaO}$ 2004). Their widths are very similar to those of Seyfert galaxies. This similarity indicates a fundamental difference in accretion rate between these two kinds of AGNs. This accretion rate difference implies that BL Lac objects do not have the same origin as Seyfert galaxies have an accretion disk origin of the optical variability. Mrk 501 has a featureless continuum, and then its optical IDVs do not have the accretion disk origin.

The variability timescales were defined in different ways. The doubling timescale is usually used to estimate the variability timescale of the large amplitude change in gamma rays (e.g., Fan et al. 1999). A fast variability in the VHE gamma rays shows doubling times $\sim 15$ minutes for Mrk 501 (Ahnen et al. 2016). But the fastest variability observed on Mrk 501 at VHE is $\sim 2$ minutes (Albert et al. 2007). These VHE gamma rays are from the relativistic jets. These variability timescales of $\sim 2-15$ minutes could give upper limits to the diameter sizes of gamma-ray emitting regions, $D_{\gamma} \lesssim c \Delta t_{\min }^{\mathrm{ob}} \delta /(1+z)$, where $c$ is the speed of light, $\Delta t_{\text {min }}^{\mathrm{ob}}$ is the minimum variability timescale observed, and $\delta$ is the corresponding Doppler factor. In general, the lower energy bands will show the smaller variability amplitudes for Mrk 501 (Ahnen et al. 2016). The early common definition of the variability timescale is $\tau=F /|\Delta F / \Delta t|$, and the more conservative approach is $\tau=|\Delta t / \Delta \ln F|$, where $F$ is the flux and $\Delta F$ is the variability amplitude on the timescale $\Delta t$ (e.g., Wagner \& Witzel 1995). These definitions were used to the variability with a large amplitude change, e.g., $\gtrsim 0.1 \mathrm{mag}$. Another choice is the interval between the adjacent local minima at the adjacent valleys in the light curve, respectively (i.e., the flare duration), or two times the interval between the adjacent local minimum and maximum at the adjacent valley and peak, respectively, if the flare is not complete. As a shock passes through the emitting region (knot or blob) in the jet, this passage will generate a flare with the duration comparable to the passing timescale of the shock. In the case, the variability timescale corresponds to the flare duration, i.e., the interval between the adjacent local 
minima at the adjacent valleys in the light curve. Another possibility is that as the blob-like emitting region becomes optical thin, it generates a flare with a duration about equal to the light crossing time of the emitting region. These definitions should locate by hand the corresponding data points in the short-term light curve.

The minimum timescale was determined by hand for the short-term light curve (e.g., Xie et al. 1990, 1991a, b, 1992, 1999, 2001, 2002c, 2005a). In an analogous fashion, we searched possible flares in the light curves on 2010 May 15 and 17. There is a darkening in the light curve on 2010 May 15 (see Figure 6). This darkening consists of 11 data points, has $\Delta R=0.030 \pm 0.005 \mathrm{mag}$ and lasts for 26.7 minutes. The corresponding rate of the change is $0.067 \mathrm{mag} / \mathrm{hr}$. The 11 data points can be fitted linearly with a Pearson's correlation coefficient $r=0.945$ at the confidence level of more than 99.99 per cent. Thus, the random fluctuation origin of this darkening can be eliminated at a high confidence level. There seems no significant adjacent rising phase before this darkening (see Figure 6). We do not get the total duration of the flare from the sum of the rising and darkening timescales. The two times of the darkening timescale may be assumed as a representative value for the duration, 0.89 hours. According to the necessary condition of optical variability $\Delta m>5 \sigma$ used in Xie et al. (1990, 1992, 2004), an IDV might be indicated for this flare, because the uncertainty $\sigma$ does not take into account the systematic uncertainty. The variability amplitude can be calculated by $A m p=\sqrt{\left(A_{\max }-A_{\min }\right)^{2}-2 \sigma^{2}}\left(\right.$ Heidt \& Wagner 1996), where $A_{\max }$ and $A_{\min }$ are the maximum and minimum magnitudes of the light curve being considered, respectively, and $\sigma$ is the measurement error. There are $A m p=4.3 \% \pm 0.7 \%$ for the light curve on 2010 May 15, and $A m p=2.9 \% \pm 0.7 \%$ for the flare mentioned above. The auto-correlation function method could search for the characteristic timescale of the large amplitude variability in the long-term light curve (e.g., Netzer et al. 1996; Giveon et al. 1999; Liu et al. 2008). The $F$ test and the ANOVA test indicate the IDV in the light curve on 2010 May 15, but 
cannot give the IDV details, e.g, timescale. The structure function (SF), introduced by Simonetti et al. (1985), has been employed to quantify the characteristic timescale for a light curve with confirmed variability (e.g., meeting some tests) (e.g., Abdo et al. 2010b; Dai et al. 2015). The first-order SF is defined as

$$
S F^{(1)}(\Delta t)=\frac{1}{N} \sum_{i=1}^{N}\left[m\left(t_{i}\right)-m\left(t_{i}+\Delta t\right)\right]^{2},
$$

where $m(t)$ is the magnitude at time $t$, and $\Delta t$ is the time separation. The characteristic timescale in a light curve is indicated by a local maximum of the SF. The first local maximum was used, i.e., the one with the shortest time, in the case of multiple local maxima in the SF (e.g., Dai et al. 2015). Figure 7 displays the first-order SF for the light curve on 2010 May 15. However, the SF cannot give "a real break or peak" (a large break or peak), i.e., a robust variability characteristic timescale. The SF is not able to determine the variability timescale of the micro-amplitude flare shown in Figure 6. The SF increases with the timescale, which implies that there is more power of variability at the longer timescales (which is a typical characteristic in blazars and AGNs in general).

The IDV is an intrinsic phenomenon and tightly constrains the sizes of the emitting regions in blazars (Wagner \& Witzel 1995). The timescales of variations in the optical band might have an underlying connection with the black hole masses of the central engines in blazars. The minimum timescales of variability were used to determine the masses of the central black holes in AGNs (e.g., Abramowicz et al. 1982; Miller et al. 1989; Xie et al. 2002a, 2005b; Liu \& Bai 2015). Abramowicz et al. (1982) and Xie et al. (2002a) determined the black hole masses $M_{\bullet}$ for non-blazar-like AGNs or some AGNs with a weaker blazar emission component in fluxes relative to an accretion disk emission component, based on the assumption that an accretion disk is surrounding a supermassive black hole, and the optical flux variations are from the accretion disk. Liu \& Bai (2015) proposed a sophisticated model to constrain $M_{\bullet}$ using the rapid variations for blazars. 
The model is suitable to constrain $M_{\bullet}$ in blazars using the minimum timescale $\Delta t_{\text {min }}^{\text {ob }}$ of variations of the beamed emission from the relativistic jets. Liu \& Bai (2015) gave

$$
\begin{aligned}
& M_{\bullet} \lesssim 5.09 \times 10^{4} \frac{\delta \Delta t_{\mathrm{min}}^{\mathrm{ob}}}{1+z} M_{\odot} \quad(j \sim 1), \\
& M_{\bullet} \lesssim 1.70 \times 10^{4} \frac{\delta \Delta t_{\min }^{\mathrm{ob}}}{1+z} M_{\odot} \quad(j=0),
\end{aligned}
$$

where $\Delta t_{\text {min }}^{\mathrm{ob}}$ is in units of seconds, and $j=J / J_{\max }$ is the dimensionless spin parameter of a black hole with the maximum possible angular momentum $J_{\max }=G M_{\bullet}^{2} / c$ and $G$ being the gravitational constant. For the light curve of Mrk 501 on 2010 May 15, the optical IDV has a darkening timescale of $\tau_{\mathrm{d}}=26.7$ minutes with a micro-amplitude of $\Delta m=0.03$ mag. We made the assumption of considering the duration of 0.89 hours, two times $\tau_{\mathrm{d}}$, as a representative value for the variability timescale to be used in formulae (3a) and (3b). Other studies used other prescriptions to estimate a variability timescale (like flux-doubling times, or characteristic times in SF), which cannot be used with the optical data reported in this paper. The optical- $\gamma$-ray emission is mostly the Doppler boosted emission of jets for $\gamma$-ray blazars (Ghisellini et al. 1998). A value of $\delta \sim 10$ was adopted for GeV gamma-ray blazars (Ghisellini et al. 2010). As in Liu \& Bai (2015),$\delta=10$ is taken to estimate $M$ • with formulas (3) for Mrk 501. In the case of $\Delta t_{\text {min }}^{\mathrm{ob}}=0.89$ hours, we have $M_{\bullet} \lesssim 10^{9.20} M_{\odot}$ for the Kerr black hole [formula (3a)] and $M_{\bullet} \lesssim 10^{8.72} M_{\odot}$ for the Schwarzchild black hole [formula (3b)]. The flare duration of 0.89 hours gives $M_{\bullet} \lesssim 10^{8.72}-10^{9.20} M_{\odot}$. Barth et al. (2003) and Falomo et al. (2002) measured the stellar velocity dispersion and estimated $M_{\bullet}=10^{9.21 \pm 0.13} M_{\odot}$ and $M_{\bullet}=10^{8.93 \pm 0.21} M_{\odot}$, respectively, by the black hole mass-stellar velocity dispersion relation. Ghisellini et al. (2010) reported a mass $M_{\bullet}=10^{8.84} M_{\odot}$. The mass upper limit of $M_{\bullet} \lesssim 10^{9.20} M_{\odot}$ is basically consistent with the mass estimates $M_{\bullet}=10^{8.93 \pm 0.21} M_{\odot}, 10^{9.21 \pm 0.13} M_{\odot}$, and $10^{8.84} M_{\odot}$. Rieger \& Mannheim (2003) showed the large uncertainties in the determination of the black hole mass of Mrk 501, ranging from $\sim 10^{7.8} M_{\odot}$ to $10^{8.7} M_{\odot}$, which are also consistent 
with the mass upper limits. We made the assumption that IDV is tightly connected to the black hole, in order to set constraints to the black hole mass using the method proposed in Liu \& Bai (2015). The derived upper bounds indicate that these two hypotheses are consistent with the measurements/data. The presence of systematic uncertainties caused by poor weather conditions, telescope tracking inaccuracies, etc, will mask the optical IDV finding for Mrk 501. Based on the method used in Du et al. (2014), we estimated the systematic uncertainty from the standard deviation of the residuals, which were obtained by subtracting the median-smoothed light curve from the original light curve. This gives a systematic uncertainty $\approx 0.007$ on 2010 May 15 . So, the IDV on 2010 May 15 is not a very robust claim because of the presence of a systematic uncertainty at the level of $\sim 1 \%$ that may affect the flux variations that were measured, which are at the level of $\sim 3 \%$. Higher accuracy observations with large aperture telescopes are needed in future.

We are grateful to the anonymous referee for constructive comments leading to significant improvement of this paper. We thank the financial supports of the National Natural Science Foundation of China (NSFC; grants No. 11273052 and U1431228), and the Youth Innovation Promotion Association, CAS. We also acknowledge the support of the staff of the Lijiang $2.4 \mathrm{~m}$ telescope. Funding for this telescope has been provided by CAS and the People's Government of Yunnan Province. 


\section{REFERENCES}

Abdo, A. A., Ackermann, M., Agudo, I., et al. 2010a, ApJ, 716, 30

Abdo, A. A., Ackermann, M., Ajello, M., et al. 2010b, ApJ, 722, 520

Abdo, A. A., Ackermann, M., Ajello, M., et al. 2011, ApJ, 727, 129

Abramowicz, M. A., \& Nobili, L. 1982, Nature, 300, 506

Agarwal, A., Gupta, A. C., Bachev, R., et al. 2016, MNRAS, 455, 680

Aharonian, F., Akhperjanian, A. G., Barrio, J. A., et al. 1999a, A\&A, 349, 29A

Aharonian, F., Akhperjanian, A. G., Barrio, J. A., et al. 1999b, A\&A, 342, 69A

Aharonian, F., Akhperjanian, A. G., Barrio, J. A., et al. 2001, ApJ, 546, 898

Aharonian, F., Akhperjanian, A. G., Bazer-Bachi, A. R., et al. 2007, ApJ, 664, L71

Ahnen, M. L., Ansoldi, S., Antonelli, L. A., et al. 2016, A\&A, accepted, arXiv: 1612.09472

Albert, J., Aliu, E., Anderhub, H., et al. 2007, ApJ, 669, 862

Aleksic, J., Ansoldi, S., Antonelli, L. A., et al. 2015, A\&A, 573, 50

Agarwal, A., \& Gupta, A. C. 2015, MNRAS, 450, 541

Bai, J. M., Xie, G. Z., Li, K. H., et al. 1998, A\&AS, 132, 83B

Barth, A. J., Ho, L. C., \& Sargent, W. L. W. 2003, ApJ, 583, 134

Blandfor, R., D., \& Königl, A. 1979, ApH, 232, 34

Böttcher, M. 2007, Ap\&SS, 309, 95

Camenzind, M., \& Krockenberger, M. 1992, A\&A, 255, 59 
Cao, X. W. 2002, ApJ, 570, L13

Cao, X. W. 2004, ApJ, 609, 80

Cao, X. W., \& Jiang, D. R. 1999, MNRAS, 1999, 307, 802

Catanese, M., Bradbury, S. M., Breslin, A. C., et al. 1997, ApJ, 487, 143

Catanese, M., \& Sambruna, R. M. 2000, ApJL, 534, L39

Celotti, A., Padovani, P., \& Ghisellini, G. 1997, MNRAS, 286, 415

Ciprini, S., Tosti, G., Raiteri, C. M., et al. 2003, A\&A, 400, 487

Ciprini, S., Takalo, L. O., Tosti, G., et al. 2007, A\&A, 467, 465

Dai, B. Z., Zeng, W., Jiang, Z. J., et al. 2015, ApJS, 218, 18

de Diego, J. A., Dultzin-Hacyan, D. Ramírez, A., \& Benítez, E. 1998, ApJ, 501, 69

de Diego, J. A. 2010, AJ, 139, 1269

Dermer, C. D., \& Schlickeiser, R. 1993, ApJ, 416, 458

Du, P., Hu, C., Lu, K. X., et al. 2014, ApJ, 782, 45

Falomo, R., Kotilainen, J. K., \& Treves, A. 2002, ApJ, 569, L35

Fan, J. H. 2005, ChJAA, 5, 213

Fan, J. H., Kurtanidze, O., Liu, Y., et al. 2014, ApJS, 213, 26

Fan, J. H., Xie, G. Z., \& Bacon, R. 1999, A\&AS, 136, 13

Fan, J. H., Zhang, Y. W., Qain, B. C., et al. 2009, ApJS, 181, 466

Fiorucci, M., \& Tosti, G. 1996, A\&AS, 116, 403 
Ghisellini, G., Celotti, A., Fossati, G., et al. 1998, MNRAS, 301, 451

Ghisellini, G., Tavecchio, F., Foschini, L., et al. 2010, MNRAS, 402, 497

Giveon, U., Maoz, D., Kaspi, S., Netzer, H., \& Smith, P. S. 1999, MNRAS, 306, 637

Gopal-Krishna \& Wiita, P. J. 1992, A\&A, 259, 109

Goyal, A., Wiita, P. J., Anupama, G. C., et al. 2012, A\&A, 544, 37

Goyal, A., Stawarz, Ł., Ostrowski, M., et al. 2017, ApJ, 837, 127

Gupta, A. C., Banerjee, D. P. K., Ashok, N. M., \& Joshi, U. C. 2004, A\&A, 422, 505

Gupta, A. C., Fan, J. H., Bai, J. M., et al. 2008a, ApJ, 135, 1384

Gupta, A. C., Deng, W. G., Joshi, U. C., Bai, J. M., \& Lee, M. G. 2008b, NewA, 13, 375

Gupta, S. P., Pandey, U.S., Singh, K., et al, 2012, NewA, 17, 8

Heidt, J., \& Wagner, S. J., 1996, A\&A, 305, 42

Hu, S. M., Chen, X., Guo, D. F., Jiang, Y. G. \& Li, K. 2014, MNRAS, 443, 2940

Impiombato, D., Covino, S., Treves, A., et al. 2011, ApJS, 192, 12

Jang, M., \& Miller, H. R. 1997, AJ, 114, 565

Joshi, R., Chand, H., Gupta, A. C., \& Wiita, P. J. 2011, MNRAS, 412, 2717

Konopelko, A., Mastichiadis, A., \& Kirk, J. 2003, ApJ, 597, 851

Liu, H. T., Bai, J. M., Zhao, X. H., \& Ma, L. 2008, ApJ, 677, 884

Liu, H. T., \& Bai, J. M. 2015, AJ, 149, 191

Marscher, A. P. 2014, ApJ, 780, 87 
Miller, H. R., Carini, M. T., \& Goodrich, B. D. 1989, Nature, 337, 627

Narayan, R., \& Piran, T. 2012, MNRAS, 420, 604

Neronov, A., Semikoz, D., \& Taylor, A. M. 2012, A\&A, 541, 31

Netzer, H., Heller, A., Loinger, F., et al. 1996, MNRAS, 279, 429

Nilsson, K., Pasanen, M., Takalo, L. O., Lindfors, E., Berdyugin, A., Ciprini, S., \& Pforr, J. 2007, A\&A, 475, 199

Pian, E., Romano, P, Treves, A., et al. 2007, ApJ, 664, 106

Quinn, J., Akerlof, C. W., Biller, S., et al. 1996, ApJL, 456, L83

Rieger, F. M., \& Mannheim, K. 2003, A\&A, 397, 121

Rodig, C., Burkart, T., Elbracht, O., \& Spanier, F. 2009, A\&A, 501, 925

Romero, G. E., Cellone, S. A., \& Combi, J. A. 1999, A\&AS, 135, 477

Saito, S., Stawarz, Ł., Tanaka, Y. T., et al. 2015, ApJ, 809, 171

Samuelson, F. W., Biller, S. D., Bond, I. H., et al. 1998, ApJ, 501, L17

Shukla, A., Chitnis, V. R., Singh, B. B., et al. 2015, ApJ, 798, 2

Simonetti, J. H., Cordes, J. M., \& Heeschen, D. S. 1985, ApJ, 296, 46

Stickel, M., Fried, J. M., \& Kuehr, H. 1993, A\&AS, 98, 393

Subramanian, P., Shukla, A., \& Becker, P. A. 2012, MNRAS, 423, 1707

Ulrich, M. H., Maraschi, L., \& Urry, C. M. 1997, ARA\&A, 35, 445

Urry, C. M., \& Padovani, P. 1995, PASP, 107, 803 
Villata, M., Raiteri, C. M., Lanteri, L., et al. 1998, A\&AS, 130, 305

Wagner, S. J., \& Witzel, A. 1995, ARA\&A, 33, 163

Wierzcholska, A., Ostrowski, M., Stawarz, L., Wagner, S., \& Hauser, M. 2015, A\&A, 573, 69

Xie, G. Z., Li, K. H., Bai, J. M., et al. 2001, ApJ, 548, 200

Xie, G. Z., Li, K. H., Cheng, F. Z., et al. 1990, A\&A, 229, 329

Xie, G. Z., Li, K. H., Cheng, F. Z., et al. 1991a, A\&AS, 87, 461

Xie, G. Z., Li, K. H., Liu, F. K., et al. 1992, ApJS, 80, 683

Xie, G. Z., Li, K. H., Zhang, X., Bai, J. M., \& Liu, W. W. 1999, ApJ, 522, 846

Xie, G. Z., Liang, E. W., Xie, Z. H., \& Dai, B. Z. 2002a, AJ, 123, 2352

Xie, G. Z., Liang, E. W., Zhou, S. B., et al. 2002b, MNRAS, 334, 459

Xie, G. Z., Liu, F. K., Liu, B. F., et al. 1991b, AJ, 101, 71

Xie, G. Z., Liu, H. T., Cha, G. W., et al. 2005a, AJ, 130, 2506

Xie, G. Z., Ma, L., Zhou, S. B., Chen, L. E., \& Xie, Z. H. 2005b, PASJ, 57, 183

Xie, G. Z., Zhou, S. B., Dai, B. Z., et al. 2002c, MNRAS, 329, 689

Xie, G. Z., Zhou, S. B., Li, K. H., Dai, H., Chen, L. E., \& Ma, L. 2004, MNRAS, 348, 831

Xiong, D. R., Zhang, H. J., Zhang, X., et al. 2015, ApJS, 222, 24

Xu, Y. D., Cao, X. W., \& Wu, Q. W. 2009, ApJ, 694, L107

Zhang, X., Zhang, L., Zhao, G., et al. 2008, AJ, 128, 1929 
Zhang, X., Zheng, Y. G., Zhang, H. J., \& Hu, S. M. 2008, ApJS, 174, 111 
Table 1. Observation log and results of IDV observations of Mrk 501

\begin{tabular}{|c|c|c|c|c|c|c|c|c|}
\hline $\begin{array}{l}\text { Date } \\
(1)\end{array}$ & $\begin{array}{c}\text { Filters } \\
(2)\end{array}$ & $\begin{array}{l}\mathrm{N} \\
(3)\end{array}$ & $\begin{array}{c}\sigma(\operatorname{star} 1-\operatorname{star} 6) \\
(4)\end{array}$ & $\begin{array}{c}F \\
(5)\end{array}$ & $\begin{array}{c}F(99) \\
(6)\end{array}$ & $\begin{array}{c}\text { ANOVA } \\
(7)\end{array}$ & $\begin{array}{c}\text { ANOVA(99) } \\
(8)\end{array}$ & $\begin{array}{c}\text { Telescope } \\
\text { (9) }\end{array}$ \\
\hline \multirow[t]{4}{*}{2005 Apr 05} & B & 4 & 0.074 & & & & & 1.02 \\
\hline & I & 5 & 0.015 & & & & & 1.02 \\
\hline & $\mathrm{R}$ & 4 & 0.01 & & & & & 1.02 \\
\hline & $\mathrm{V}$ & 5 & 0.015 & & & & & 1.02 \\
\hline \multirow[t]{4}{*}{2005 Apr 06} & B & 1 & & & & & & 1.02 \\
\hline & I & 9 & 0.013 & 0.23 & 6.03 & 3.37 & 10.92 & 1.02 \\
\hline & $\mathrm{R}$ & 10 & 0.018 & 0.19 & 5.35 & 0.41 & 9.55 & 1.02 \\
\hline & $\mathrm{V}$ & 9 & 0.033 & 0.02 & 6.03 & 0.29 & 10.92 & 1.02 \\
\hline \multirow[t]{2}{*}{2006 Apr 03} & I & 4 & 0.054 & & & & & 1.02 \\
\hline & $\mathrm{R}$ & 4 & 0.024 & & & & & 1.02 \\
\hline \multirow[t]{2}{*}{2007 Mar 27} & I & 39 & 0.012 & 0.48 & 2.16 & 3.45 & 2.96 & 1.02 \\
\hline & V & 28 & 0.076 & 0.03 & 2.51 & 1.13 & 3.63 & 1.02 \\
\hline \multirow[t]{2}{*}{2007 Mar 28} & I & 5 & 0.008 & & & & & 1.02 \\
\hline & $\mathrm{V}$ & 3 & 0.01 & & & & & 1.02 \\
\hline \multirow[t]{2}{*}{2007 Mar 29} & I & 8 & 0.005 & & & & & 1.02 \\
\hline & $\mathrm{V}$ & 4 & 0.023 & & & & & 1.02 \\
\hline \multirow[t]{2}{*}{2007 Mar 30} & I & 8 & 0.006 & & & & & 1.02 \\
\hline & $\mathrm{V}$ & 5 & 0.027 & & & & & 1.02 \\
\hline 2007 Apr 15 & I & 13 & 0.015 & 0.46 & 4.16 & 2.81 & 6.99 & 1.02 \\
\hline \multirow[t]{3}{*}{2007 Apr 22} & B & 2 & 0.013 & & & & & 1.02 \\
\hline & I & 10 & 0.006 & 0.63 & 5.35 & 0.31 & 9.55 & 1.02 \\
\hline & $\mathrm{V}$ & 10 & 0.04 & 0.22 & 5.35 & 1.54 & 9.55 & 1.02 \\
\hline 2007 Apr 23 & I & 4 & 0.005 & & & & & 1.02 \\
\hline \multirow[t]{2}{*}{2007 Apr 24} & I & 15 & 0.007 & 2.42 & 3.7 & 0.54 & 5.99 & 1.02 \\
\hline & $\mathrm{V}$ & 14 & 0.052 & 0.21 & 3.91 & 6.22 & 6.55 & 1.02 \\
\hline 2007 May 08 & I & 10 & 0.012 & 1.21 & 5.35 & 0.42 & 9.55 & 1.02 \\
\hline \multirow[t]{3}{*}{2007 May 09} & B & 1 & & & & & & 1.02 \\
\hline & I & 9 & 0.009 & 0.58 & 6.03 & 1.68 & 10.92 & 1.02 \\
\hline & $\mathrm{V}$ & 5 & 0.032 & & & & & 1.02 \\
\hline 2008 May 06 & I & 4 & 0.037 & & & & & 1.02 \\
\hline 2008 May 07 & I & 87 & 0.009 & 1.05 & 1.69 & 1.81 & 2.08 & 1.02 \\
\hline
\end{tabular}


Table 1-Continued

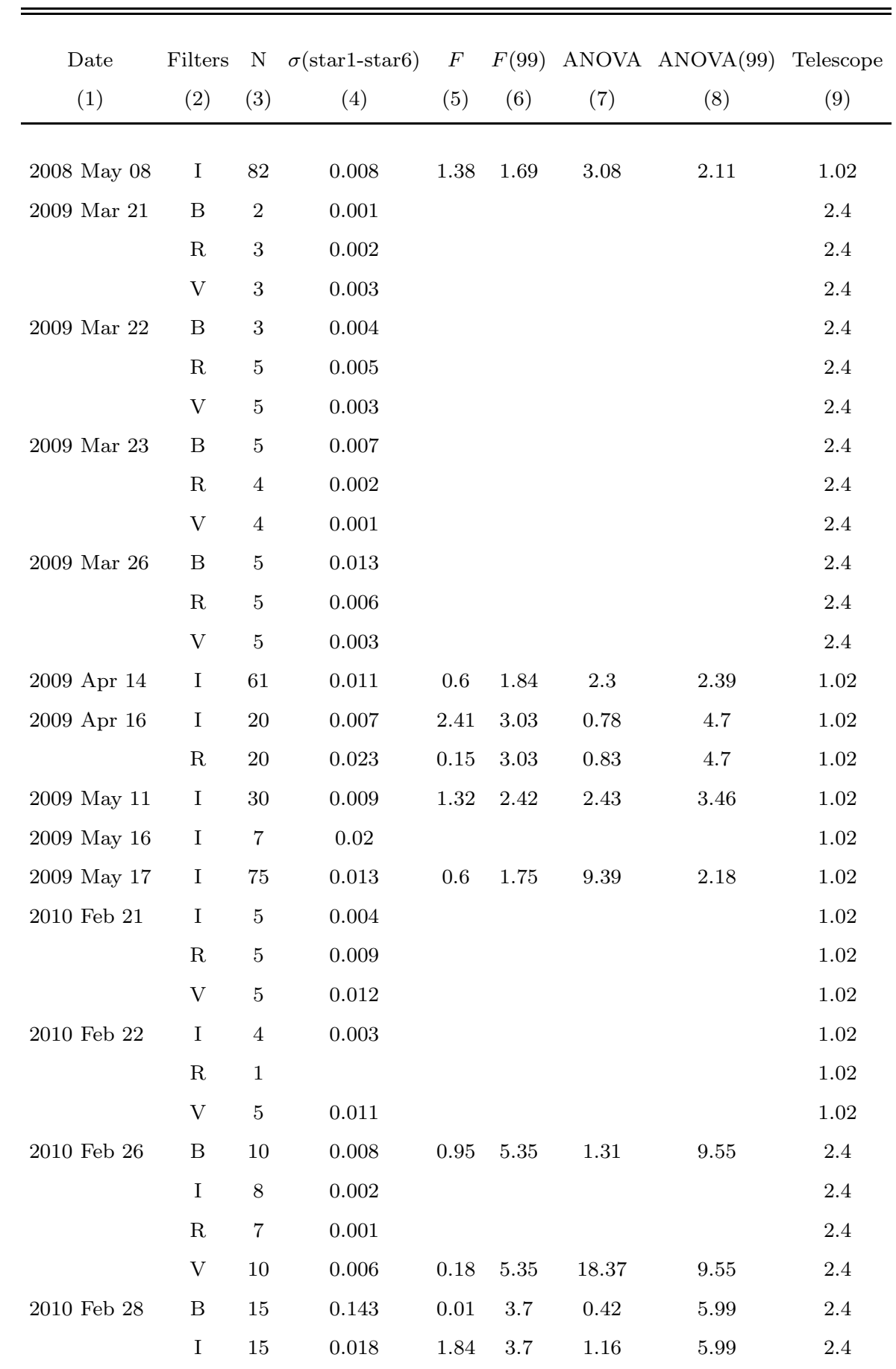


Table 1-Continued

\begin{tabular}{|c|c|c|c|c|c|c|c|c|}
\hline $\begin{array}{l}\text { Date } \\
(1)\end{array}$ & $\begin{array}{c}\text { Filters } \\
(2)\end{array}$ & $\begin{array}{l}\mathrm{N} \\
(3)\end{array}$ & $\begin{array}{c}\sigma(\operatorname{star} 1-\operatorname{star} 6) \\
(4)\end{array}$ & $\begin{array}{c}F \\
(5)\end{array}$ & $\begin{array}{c}F(99) \\
(6)\end{array}$ & $\begin{array}{c}\text { ANOVA } \\
(7)\end{array}$ & $\begin{array}{c}\operatorname{ANOVA}(99) \\
(8)\end{array}$ & $\begin{array}{c}\text { Telescope } \\
\text { (9) }\end{array}$ \\
\hline \multirow{6}{*}{2010 Mar 11} & $\mathrm{R}$ & 15 & 0.015 & 0.17 & 3.7 & 1.75 & 5.99 & 2.4 \\
\hline & $\mathrm{V}$ & 15 & 0.025 & 0.22 & 3.7 & 1.9 & 5.99 & 2.4 \\
\hline & B & 1 & & & & & & 2.4 \\
\hline & I & 1 & & & & & & 2.4 \\
\hline & $\mathrm{R}$ & 1 & & & & & & 2.4 \\
\hline & $\mathrm{V}$ & 1 & & & & & & 2.4 \\
\hline \multirow[t]{2}{*}{2010 May 04} & $\mathrm{R}$ & 19 & 0.007 & 0.92 & 3.13 & 0.95 & 4.86 & 1.02 \\
\hline & V & 18 & 0.01 & 0.37 & 3.24 & 0.77 & 5.06 & 1.02 \\
\hline 2010 May 15 & $\mathrm{R}$ & 88 & 0.005 & 5.14 & 1.69 & 4.37 & 2.08 & 1.02 \\
\hline 2010 May 16 & $\mathrm{R}$ & 88 & 0.007 & 1.03 & 1.69 & 1.67 & 2.08 & 1.02 \\
\hline 2010 May 17 & $\mathrm{R}$ & 80 & 0.005 & 5.31 & 1.75 & 1.75 & 2.17 & 1.02 \\
\hline 2010 May 18 & $\mathrm{R}$ & 70 & 0.005 & 1.12 & 1.79 & 2.26 & 2.27 & 1.02 \\
\hline 2011 May 07 & $\mathrm{R}$ & 60 & 0.007 & 1.44 & 1.93 & 1.18 & 2.39 & 1.02 \\
\hline 2011 May 09 & $\mathrm{R}$ & 63 & 0.007 & 2.11 & 1.84 & 2.15 & 2.37 & 1.02 \\
\hline 2011 May 10 & $\mathrm{R}$ & 38 & 0.012 & 0.47 & 2.21 & 0.69 & 3.02 & 1.02 \\
\hline \multirow[t]{4}{*}{2011 Aug 21} & B & 5 & 0.046 & & & & & 1.02 \\
\hline & I & 5 & 0.013 & & & & & 1.02 \\
\hline & $\mathrm{R}$ & 5 & 0.019 & & & & & 1.02 \\
\hline & V & 5 & 0.019 & & & & & 1.02 \\
\hline \multirow[t]{4}{*}{2011 Aug 22} & B & 5 & 0.054 & & & & & 1.02 \\
\hline & I & 5 & 0.065 & & & & & 1.02 \\
\hline & $\mathrm{R}$ & 4 & 0.06 & & & & & 1.02 \\
\hline & $\mathrm{V}$ & 5 & 0.075 & & & & & 1.02 \\
\hline \multirow[t]{3}{*}{2012 Feb 27} & I & 5 & 0.009 & & & & & 1.02 \\
\hline & $\mathrm{R}$ & 5 & 0.012 & & & & & 1.02 \\
\hline & $\mathrm{V}$ & 5 & 0.021 & & & & & 1.02 \\
\hline \multirow[t]{3}{*}{2012 Feb 28} & I & 5 & 0.011 & & & & & 1.02 \\
\hline & $\mathrm{R}$ & 5 & 0.023 & & & & & 1.02 \\
\hline & $\mathrm{V}$ & 4 & 0.011 & & & & & 1.02 \\
\hline \multirow[t]{2}{*}{2012 Apr 02} & I & 4 & 0.004 & & & & & 1.02 \\
\hline & $\mathrm{R}$ & 5 & 0.026 & & & & & 1.02 \\
\hline
\end{tabular}


Table 1-Continued

\begin{tabular}{|c|c|c|c|c|c|c|c|c|}
\hline $\begin{array}{l}\text { Date } \\
(1)\end{array}$ & $\begin{array}{c}\text { Filters } \\
(2)\end{array}$ & $\begin{array}{l}\mathrm{N} \\
(3)\end{array}$ & $\begin{array}{c}\sigma(\text { star1-star6) } \\
(4)\end{array}$ & $\begin{array}{c}F \\
(5)\end{array}$ & $\begin{array}{c}F(99) \\
(6)\end{array}$ & $\begin{array}{c}\text { ANOVA } \\
(7)\end{array}$ & $\begin{array}{c}\text { ANOVA(99) } \\
(8)\end{array}$ & $\begin{array}{c}\text { Telescope } \\
\text { (9) }\end{array}$ \\
\hline \multirow{5}{*}{2012 Apr 29} & $\mathrm{~V}$ & 4 & 0.039 & & & & & 1.02 \\
\hline & B & 4 & 0.056 & & & & & 1.02 \\
\hline & I & 4 & 0.009 & & & & & 1.02 \\
\hline & $\mathrm{R}$ & 4 & 0.009 & & & & & 1.02 \\
\hline & $\mathrm{V}$ & 4 & 0.019 & & & & & 1.02 \\
\hline \multirow[t]{3}{*}{2012 May 01} & I & 5 & 0.007 & & & & & 1.02 \\
\hline & $\mathrm{R}$ & 5 & 0.005 & & & & & 1.02 \\
\hline & V & 5 & 0.028 & & & & & 1.02 \\
\hline \multirow[t]{4}{*}{2012 May 03} & B & 4 & 0.03 & & & & & 1.02 \\
\hline & I & 4 & 0.022 & & & & & 1.02 \\
\hline & $\mathrm{R}$ & 4 & 0.011 & & & & & 1.02 \\
\hline & V & 4 & 0.021 & & & & & 1.02 \\
\hline \multirow[t]{3}{*}{2012 May 11} & I & 5 & 0.011 & & & & & 1.02 \\
\hline & $\mathrm{R}$ & 5 & 0.014 & & & & & 1.02 \\
\hline & $\mathrm{V}$ & 5 & 0.025 & & & & & 1.02 \\
\hline 2012 May 13 & $\mathrm{~V}$ & 1 & & & & & & 1.02 \\
\hline 2012 May 16 & $\mathrm{R}$ & 31 & 0.02 & 0.26 & 2.39 & 1.11 & 3.4 & 1.02 \\
\hline 2012 May 17 & $\mathrm{R}$ & 26 & 0.015 & 0.58 & 2.6 & 2.13 & 3.84 & 1.02 \\
\hline
\end{tabular}

Note. — Column 1: observation dates; Column 2: observation filters; Column 3: observation numbers; Column 4: observational errors estimated by standard star 1 and star 6 ; Column 5: the $F$ values in the $F$ test for the observation data; Column 6: $F(99)$ is the critical $F$ value at a $99 \%$ confidence level; Column 7: the ANOVA values in the ANOVA test for the observation data; Column 8: $\operatorname{ANOVA}(99)$ is the critical ANOVA value at a $99 \%$ confidence level; Column 9: 1.02 and 2.4 represent $1.02 \mathrm{~m}$ and $2.4 \mathrm{~m}$ telescopes, respectively. 
Table 2. The observational data for Mrk 501

\begin{tabular}{|c|c|c|c|c|c|c|c|c|c|c|c|}
\hline \multicolumn{3}{|c|}{$B$} & \multicolumn{3}{|c|}{$I$} & \multicolumn{3}{|c|}{$R$} & \multicolumn{3}{|c|}{$V$} \\
\hline MJD - 50000 & Mag & FWHM & MJD - 50000 & Mag & FWHM & MJD - 50000 & Mag & FWHM & MJD - 50000 & Mag & FWHM \\
\hline 3465.856933 & 14.459 & 3.39 & 3465.847570 & 12.579 & 2.65 & 3465.865243 & 12.942 & 3.36 & 3465.853114 & 13.382 & 3.24 \\
\hline 3465.872859 & 14.431 & 3.39 & 3465.862686 & 12.576 & 2.66 & 3465.881111 & 12.938 & 3.27 & 3465.868264 & 13.361 & 3.15 \\
\hline 3465.887859 & 14.450 & 4.45 & 3465.878669 & 12.573 & 2.48 & 3465.895648 & 12.943 & 3.26 & 3465.883577 & 13.368 & 3.39 \\
\hline 3465.902095 & 14.457 & 3.30 & 3465.893345 & 12.586 & 2.77 & 3465.909653 & 12.942 & 3.20 & 3465.898079 & 13.368 & 3.57 \\
\hline 3466.806713 & 14.602 & 2.49 & 3465.907292 & 12.587 & 2.37 & 3466.800787 & 13.146 & 1.85 & 3465.912072 & 13.364 & 3.36 \\
\hline 4205.897373 & 14.254 & 3.29 & 3466.798473 & 12.459 & 1.69 & 3466.813588 & 13.137 & 2.55 & 3466.802917 & 13.579 & 2.31 \\
\hline 4205.900324 & 14.254 & 3.18 & 3466.811401 & 12.460 & 2.29 & 3466.822234 & 13.132 & 2.28 & 3466.815973 & 13.592 & 2.37 \\
\hline 4229.711204 & 14.478 & 1.71 & 3466.819977 & 12.467 & 2.22 & 3466.831366 & 13.146 & 2.29 & 3466.824537 & 13.583 & 2.58 \\
\hline$\cdots$ & $\cdots$ & $\cdots$ & $\cdots$ & $\cdots$ & $\cdots$ & $\cdots$ & $\cdots$ & $\cdots$ & $\cdots$ & $\ldots$ & $\ldots$ \\
\hline
\end{tabular}

Note. - This table is available in its entirety in a machine-readable form in the online journal. A portion is shown here for guidance regarding its form and content. FWHM is for the standard stars, and is in units of arc-seconds. Mag denotes magnitude. 

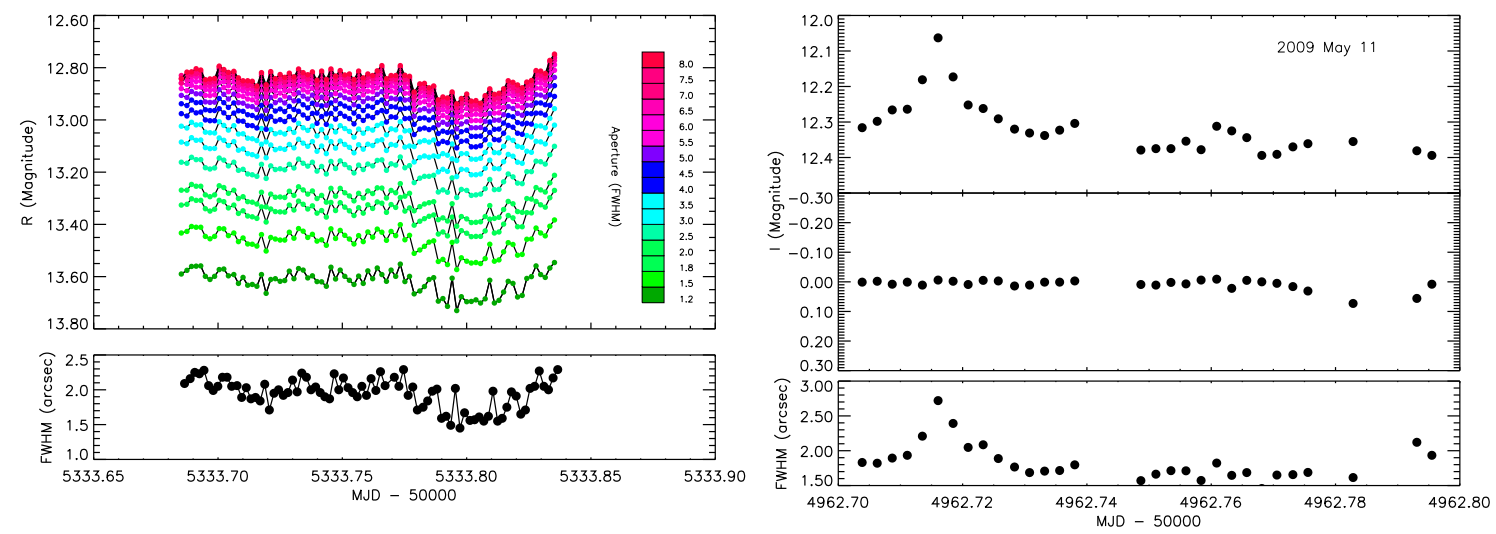

Fig. 1. - Examples of the aperture radii test for the $R$ band on 2010 May 17 and the $I$ band on 2009 May 11. In the left panels, the color bar shows the different aperture in units of FWHM, and the lower panel shows the FWHM variability. In the right panels, the top panel is the light curve as an aperture of 6.0 FWHMs is used, the middle panel is the differential variations of two comparison stars, and the bottom panel is the FWHM variability.
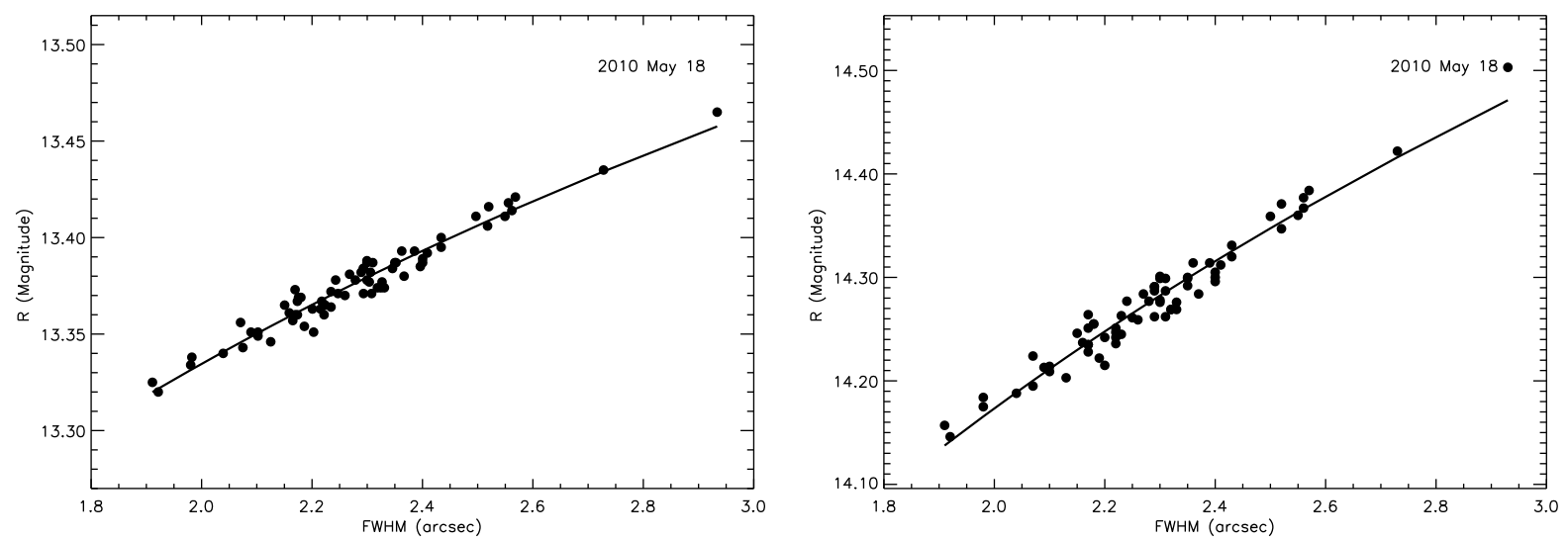

Fig. 2.- Example of seeing influence on photometric results of Mrk 501 (i.e., the FWHMmagnitude relation). The left panel denotes the observational results on 2010 may 18. The right panel shows the host-subtracted results according to Nilsson et al. (2007). Black solid circles denote the observational results, and solid lines are the fit ones. 

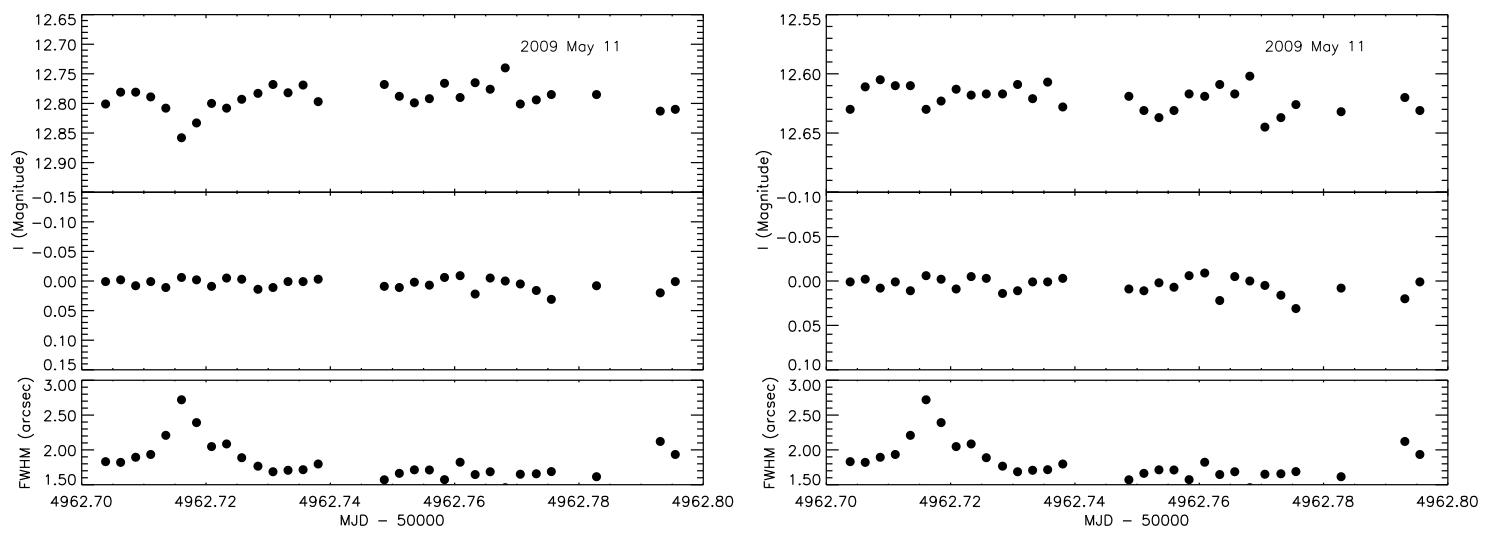

Fig. 3.- Example of the correcting seeing effect for the $I$ band on 2009 May 11. The curves are the same as in the right panel of Figure 1, except for a fixed aperture. The left panel denotes the results without correcting the seeing effect, and the right panel denotes the corrected results.
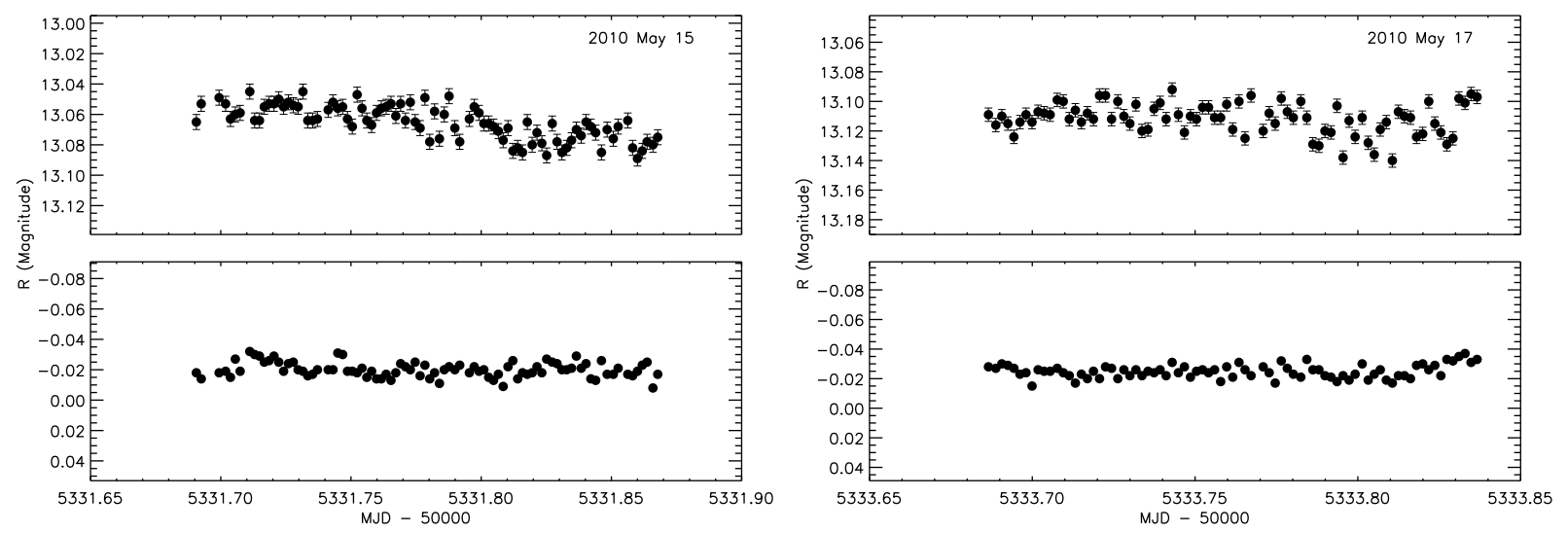

Fig. 4.- Examples of the possible IDVs for Mrk 501. For each night, the top panel is the light curve of Mrk 501, and the bottom panel is the differential variations of comparison stars 1 and 6. 

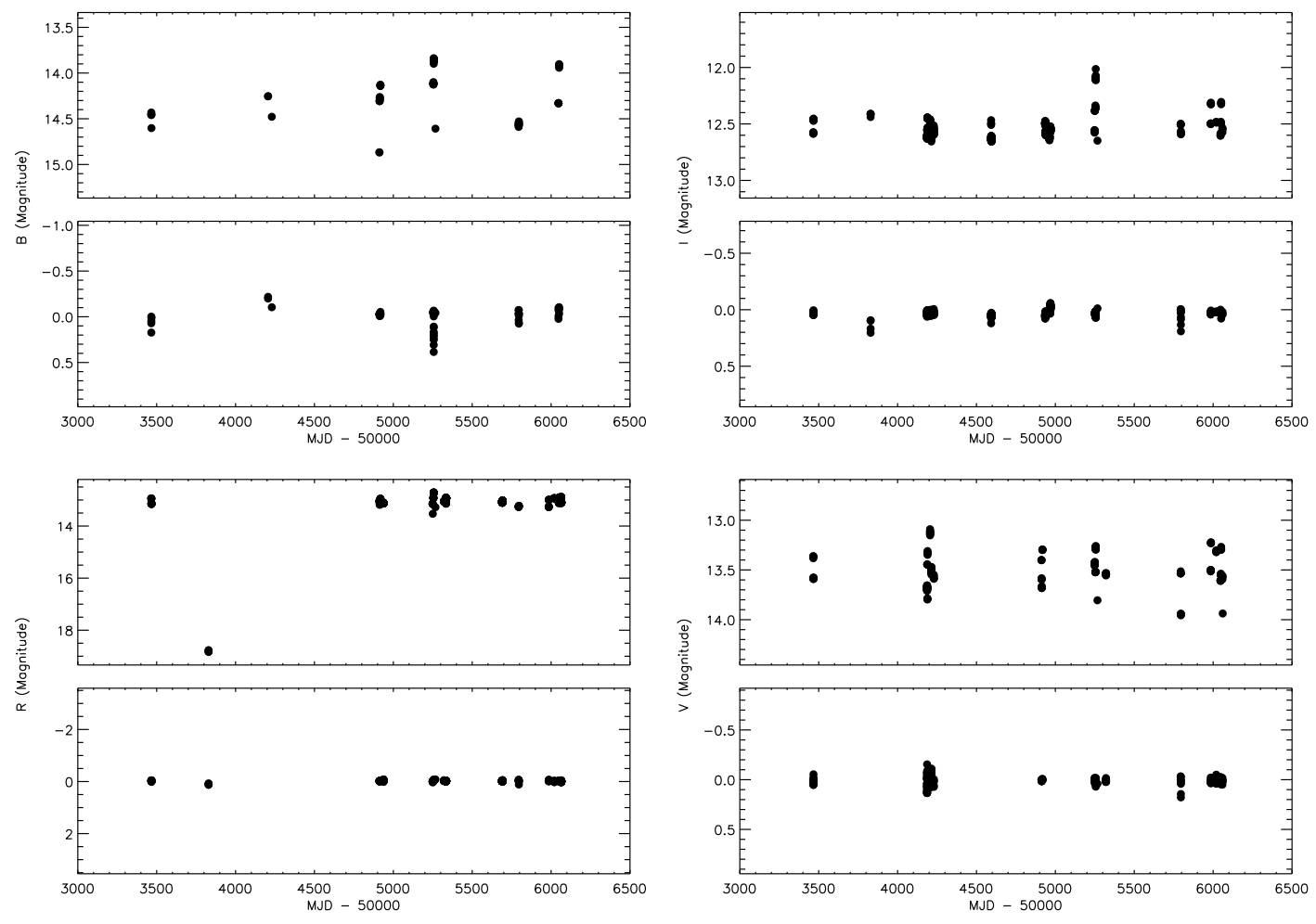

Fig. 5.- Long term light curves in the $B, I, R$, and $V$ bands for Mrk 501. For each band, the top panel is the light curve and the bottom panel is the differential variations of two comparison stars 1 and 6 . 


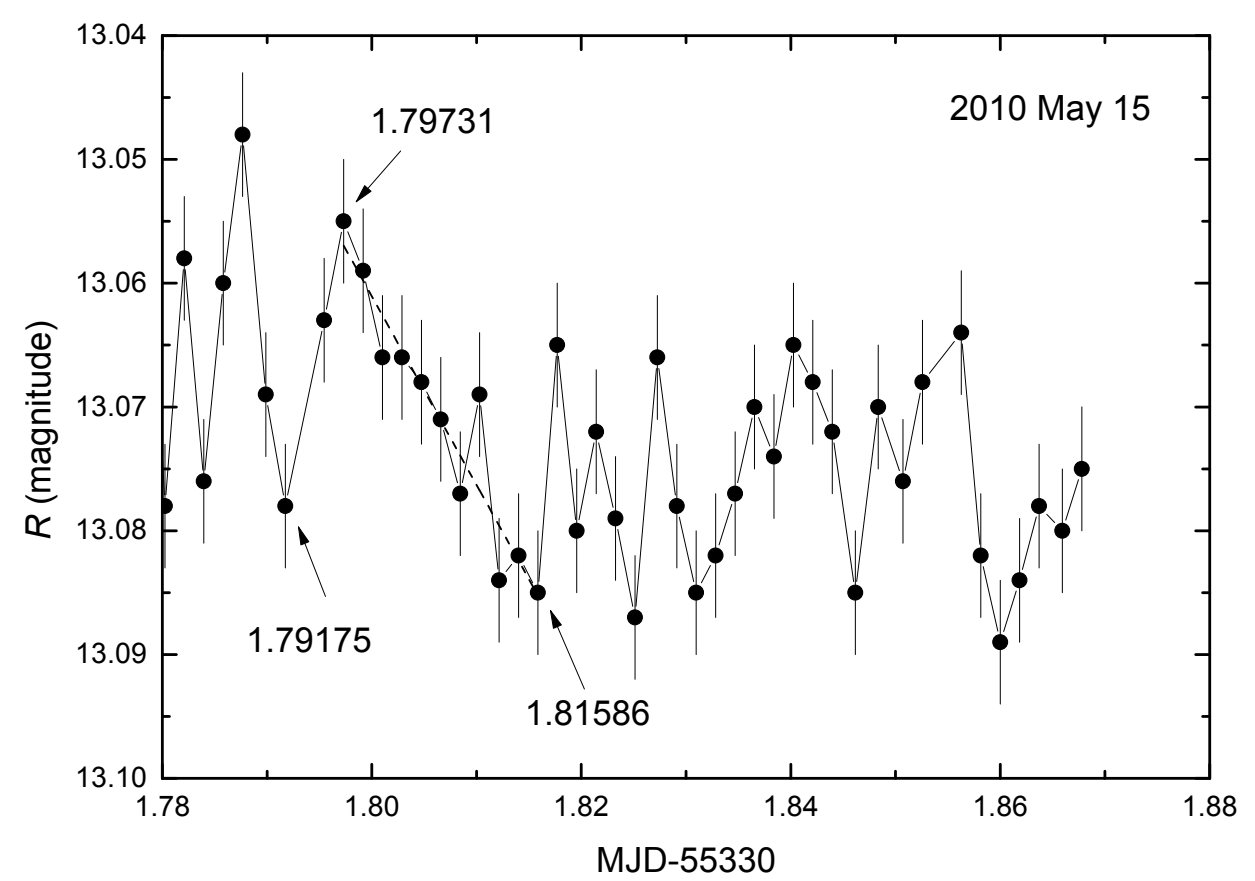

Fig. 6. - A possible IDV in one night for Mrk 501. The numbers in panel are the corresponding times of data points denoted by the arrows. The dashed line is the best linear fitting to the 11 data points with the $y$ errors $[y=10.32( \pm 0.32)+1.52( \pm 0.18) x]$. 


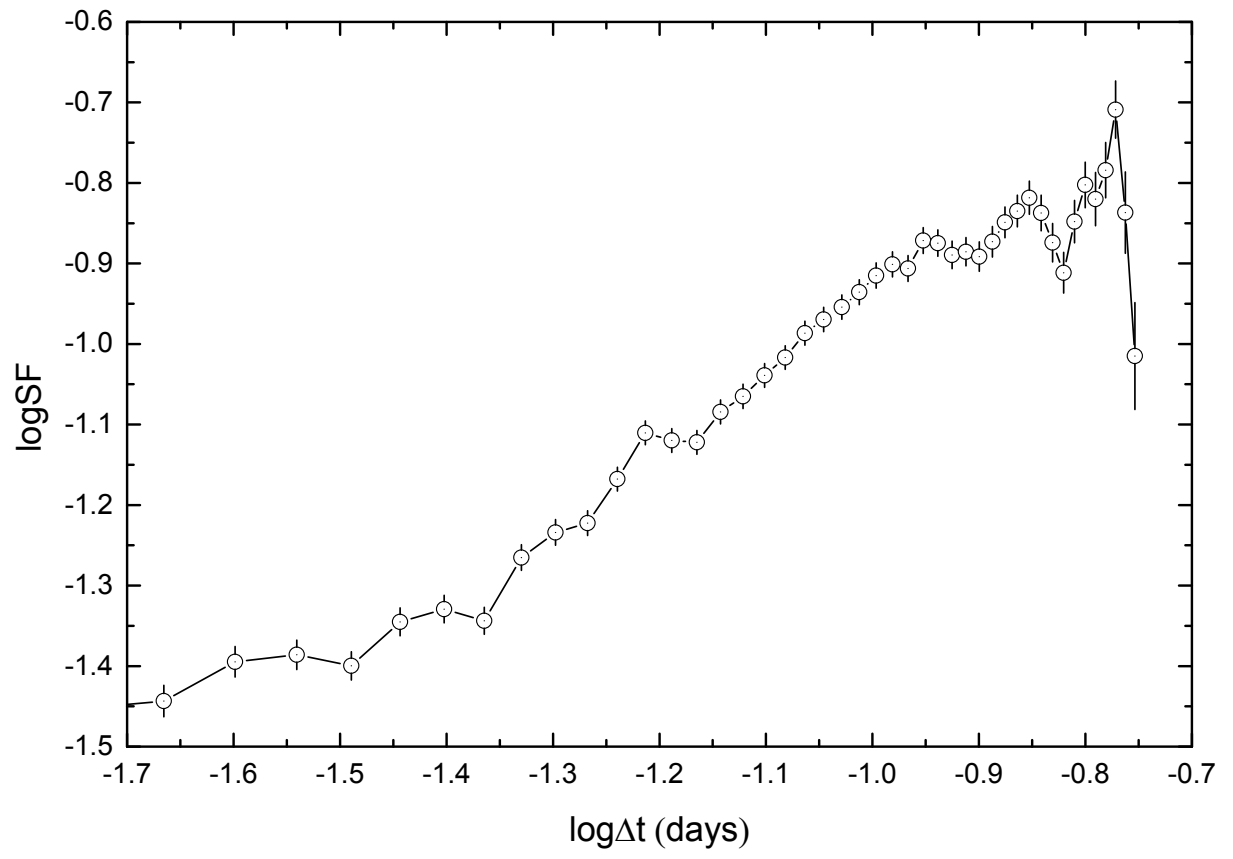

Fig. 7.- Structure Function for the light curve on 2010 May 15. 